\title{
Determination of Quinolones in Human Urine and Water Samples by Ultrasound Assisted Dispersive Liquid-Liquid Microextraction Based on Solidification of Floating Organic Droplet Followed High Performance Liquid Chromatography
}

\author{
Sen $\mathrm{Lv}^{1}$, Yiqing Sun ${ }^{1}$, Ying Yang ${ }^{1}$, Zongliang Niu ${ }^{1}$, Yingying Wen ${ }^{2 *}$ \\ ${ }^{I}$ Department of Environmental Science, School of Tropical and Laboratory Medicine, Hainan Medical University, \\ Haikou 571199, China \\ ${ }^{2}$ Laboratory of Pathogenic Biology and Immunology, School of Basic Medical Science, Hainan Medical University, \\ Haikou 571199, China
}

*Corresponding Author: Yingying Wen, Department of Environmental Science, School of Tropical and Laboratory Medicine, Hainan Medical University, Haikou 571199, China

\begin{abstract}
A ultrasound-assisted dispersive liquid-liquid microextraction based on solidification of floating organic droplet (UDLLME-SFO) combined with high performance liquid chromatography (HPLC) method was developed for the determination of quinolones $(Q N s)$ in human urine and water samples. The parameters of UDLLME-SFO procedure including sample $\mathrm{pH}$, type and volume of disperser solvent, type and volume of extraction solvent and ultrasound time were optimized. The optimal extraction conditions of UDLLME-SFO were: sample $\mathrm{pH} \mathrm{5;}$ extraction solution, $300 \mu \mathrm{L}$ 1-dodecanol; dispersion solution, $200 \mu \mathrm{L}$ methanol; ultrasound time, $10 \mathrm{~min}$. Under the optimum extraction and determination conditions, good responses for QNs were obtained in a range of $1-15 \mu \mathrm{g} / \mathrm{mL}$, with linear coefficients greater than 0.97. The recoveries of the quinolones ranged from $66 \%$ to $102 \%$. The limits of detection based on a signal-to-noise ratio of 3 was 0.03 $\mu \mathrm{g} / \mathrm{mL}$. The method was applied to the determination of QNs in human urine, tap and lake water samples with a satisfactory result. The results demonstrated that this UDLLME-SFO-HPLC method was successfully applied to determine QNs in urine and water samples.
\end{abstract}

Keywords: Dispersive liquid-liquid microextraction based on solidification of floating organic droplet; Quinlonones; Human urine; Water samples;

Abbreviations:

ACN: acetonitrile; CIP: ciprofloxacin; HPLC-UV: high performance liquid chromotagraphy-ultraviolet; LOD: limit of detection; MeOH: methanol; MOX: moxifloxacin; NOR: norfloxacin; QNs: quinolones; UDLLME-SFO: ultrasound assisted dispersive liquid-liquid microextraction based on solidification of floating organic droplet.

\section{INTRODUCTION}

Quinolones (QNs) are the most important class of synthetic antibiotics in human and veterinary medicines. They are effective to control a wide range of bacteria, both gram positive and negative as well as other bacteria and often used in the treatment of a range of illnesses. Due to the fact that its resistance in the environment can cause undesirable effects on aquatic ecosystems and human health, QNs have obtained growing attention [1,2]. Therefore, the analysis of the occurrence, distribution and risks of quinolone antibiotics became crucial to environment monitoring.

In recent years, several extraction methods have been developed for DES including solid phase extraction [1], dispersive liquid-liquid microextraction [3], liquid-liquid extraction [4], QuEChERS[5], ultrasound assisted extraction[6], stir bar sorptive extraction [7] and so on. In these extraction methods, DLLME 
Determination of Quinolones in Human Urine and Water Samples by Ultrasound Assisted Dispersive LiquidLiquid Microextraction Based on Solidification of Floating Organic Droplet Followed High Performance Liquid Chromatography

method is a novel miniaturized sample pre-treatment technique which requires smaller amounts of organic solvents and reduces the analysis cost more compared with other methods, such as LLE and SPE. The principle of DLLME was already described in our previous works[8-10]. Another mode of DLLME called dispersive liquid-liquid microextraction based on solidification of floating organic droplet (DLLME-SFO) has also been applied in various samples[10].

In this study, we propose the use of ultrasound assisted dispersive liquid-liquid microextraction based on solidification of floating organic droplet (UDLLME-SFO) for extraction of QNs from human urine and two water samples, and high performance liquid chromotagraphy-ultraviolet (HPLC-UV) for determination of QNs. To the best of our knowledge, this is the first demonstration for UDLLME-SFOHPLC-UV for QNs analysis.

\section{EXPERIMENTAL}

\subsection{Chemicals and Materials}

QNs including norfloxacin (NOR), ciprofloxacin (CIP) and moxifloxacin (MOX) were purchased from Sigma-Aldrich (Steinheim, Germany), 1-undecanol, and 1-dodecanol were also from Sigma-Aldrich (Steinheim, Germany), high performance liquid chromatography-grade methanol (MeOH) and acetonitrile (ACN) were provided by J\&K Chemical (Beijing, China). NaH2PO4, H3PO4, NaOH, and other affiliated chemicals were all obtained from Sinopharm Chemical Reagent Co. Ltd. (Shanghai, China). All solvents and chemicals were of analytical grade and used without further purification unless otherwise specified. HPLC-grade water was obtained by purifying demineralized water in a Milli-Q system (Millipore, Bedford, MA, USA), and was used throughout the work.

\subsection{Apparatus and Software}

For chromatographic separation, an Agilent 1260 HPLC system (Agilent Technologies, CA, USA), equipped with a quaternary pump, a degasser, a column compartment, and a UV detector was used. Separation was performed on a Pursuit $5 \mathrm{C} 18,5 \mu \mathrm{m}, 4.6 \mathrm{~mm} \times 250 \mathrm{~mm}$ column. The injection volume was $20 \mu \mathrm{L}$ and the ultraviolet (UV) detector was set at $290 \mathrm{~nm}$. The mobile phase consisted of (A) $0.2 \%$ acetic acid and (B) ACN with binary gradient elution at a flow rate of $1.0 \mathrm{~mL} / \mathrm{min}$. The gradient elution started with $80 \%$ (A) for $3 \mathrm{~min}$, linearly decreased to $50 \%$ (A) in $7.0 \mathrm{~min}$, then was brought back to $80 \%$ (A) in $2.0 \mathrm{~min}$ and maintained for $2.0 \mathrm{~min}$ with a total running time of $14 \mathrm{~min}$. All the samples were passed through microporous nylon filters of $0.45 \mu \mathrm{m}$ pore sizes in diameter (Pall Corporation, USA). An Ion $510 \mathrm{pH}$ meter (Ayer Rajah Crescent, Singapore) was used to monitor $\mathrm{pH}$ adjustment. A centrifuge (Xiangyi, Hunan, China) was used for sample preparation.

\subsection{Preparation of Standard and Sample}

Standard stock solution containing $1000 \mu \mathrm{g} / \mathrm{mL}$ of QNs was prepared by dissolving the required amounts of the standard in $\mathrm{MeOH}$. It was stored in a refrigerator at $4{ }^{\circ} \mathrm{C}$. Working solutions were prepared from the stock solutions by dilution with appropriate amounts of Milli-Q water.

Midstream urine sample was collected from a volunteer (22-year-old male) who had not taken QNs at all. Lake water was collected from an artificial lake located in Longhua District of Haikou City (China). Tap water was obtained in the laboratory when needed. All the water samples were passed through microporous nylon filters with the pore sizes of $0.45 \mu \mathrm{m}$ in diameter. The samples were kept under refrigeration at $4^{\circ} \mathrm{C}$ in the dark. Several aliquots from $10 \mathrm{~mL}$ filtered samples were spiked with QNs standard with different concentrations and followed by the DLLME-SFO procedure.

\subsection{UDLLME-SFO Procedure}

Briefly, $10.00 \mathrm{~mL}$ of water sample was placed in a $15 \mathrm{~mL}$ of screw-cap glass tube with conic bottom and spiked with QNs standard at $10 \mu \mathrm{g} / \mathrm{mL}$. Two hundred microliter $(200 \mu \mathrm{L})$ of $\mathrm{MeOH}$ (as disperser solvent) containing $300 \mu \mathrm{L}$ 1-dodecanol (as extraction solvent) were rapidly injected into the sample solution with a $1.00 \mathrm{~mL}$ glass syringe and the mixture was gently shaken then ultrasonicated for $10 \mathrm{~min}$. In this step, a 
Determination of Quinolones in Human Urine and Water Samples by Ultrasound Assisted Dispersive LiquidLiquid Microextraction Based on Solidification of Floating Organic Droplet Followed High Performance Liquid Chromatography

cloudy solution was formed and the QNs in the water samples was extracted into fine droplets. Then, the mixture was centrifuged for $10 \mathrm{~min}$ at $2000 \mathrm{rpm}$. After centrifugation, the fine 1-dodecanol droplets floating on the top of the tube were cooled in an ice bath. The organic solvent was solidified and transferred into a conical vial by a small medicine spoon and then melted quickly at ambient conditions. Finally, the extractant was redissolved in $1.5 \mathrm{~mL} \mathrm{MeOH}$ and analyzed by HPLC-UV. The peak area (A) of each QNs was used as the index of extraction efficiency.

\section{RESULTS AND DISCUSSION}

\subsection{Udllme-Sfo Condition Optimization}

\subsubsection{Effect of Sample Ph}

It is well known that the $\mathrm{pH}$ of the solution can affect the extraction of acidic/basic analytes due to their dissociation equilibrium. According to the literature, the acid dissociation constants of QNs were lower than 5[11]. The ionizable functional groups carboxylic (pKa,1) and nitrogen (pKa,2) are responsible for cationic, anionic, neutral and zwitterionic species according to $\mathrm{pH}$. The $\mathrm{pH}$ of sample solution was studied within the range 2.0-6.0. As shown in Fig. 1a, the peak area of NOR, CIP and MOX was the biggest when the sample $\mathrm{pH}$ was 5. This was because the neutral species of QNs were the dominant form when the sample $\mathrm{pH}$ was 5, therefore QNs was easily extracted to the 1-dodecanol phase. Finally, $\mathrm{pH} 5$ was selected as the optimum $\mathrm{pH}$ value of sample solution.

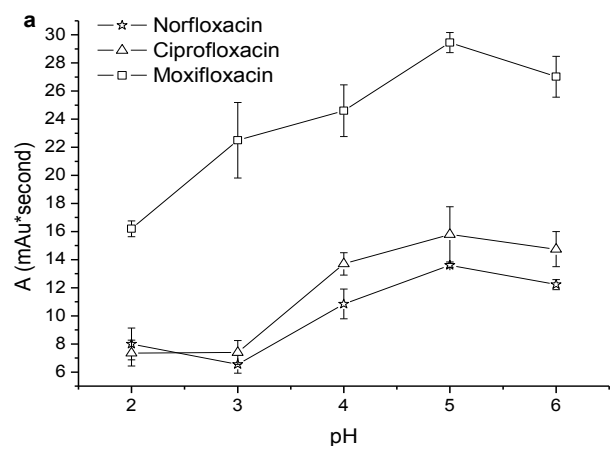

\subsubsection{Effect of Extraction Solution Type and Volume}

In UDLLME, it is very important to select an appropriate organic solvent for obtaining a satisfactory extraction. In this work, 1-undecanol and 1-dodecanol were optimized. The result showed that the extraction efficiency of 1-dodecanol was higher than the extraction efficiency of 1-undecanol, see Fig. 1b.

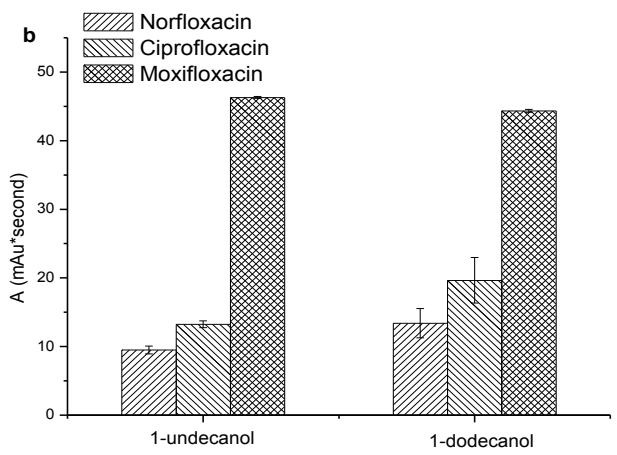

Initially, the amount of the extraction solution is also one of the important factors which affected the peak area of the QNs. Increasing the volume of extraction solution increased the recovery of QNs; however, the large volume of extraction solution decreased the concentration of QNs and therefore resulted in low extraction efficiency. The result was shown in Fig. 1c. When the extraction solution volume was $300 \mu \mathrm{L}$, the A values of QNs were the biggest. Therefore, three hundred microliter 1-dodecanol was selected as the extraction solution. 
Determination of Quinolones in Human Urine and Water Samples by Ultrasound Assisted Dispersive LiquidLiquid Microextraction Based on Solidification of Floating Organic Droplet Followed High Performance Liquid Chromatography

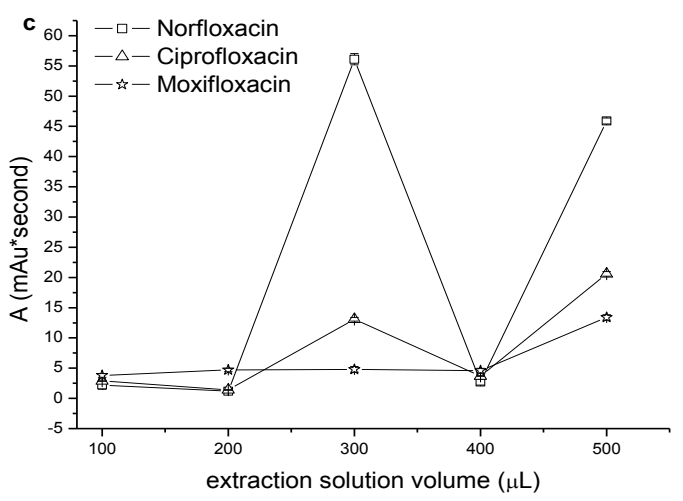

\subsubsection{Effect of Dispersion Solution Type and Volume}

Different dispersion solution will have different extraction efficiency. In this work, $\mathrm{ACN}$ and $\mathrm{MeOH}$ were optimized. The result showed that the extraction efficiency of $\mathrm{MeOH}$ was higher than the extraction efficiency of ACN, see Fig. 1d.

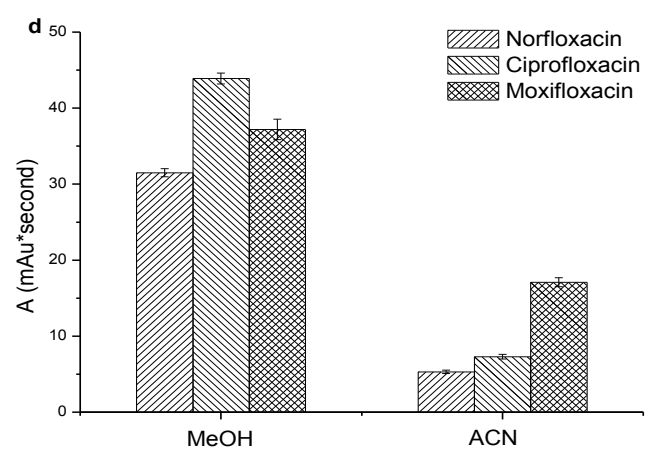

Increasing the volume of extraction solution increased the solubility of QNs, this may help QNs be extracted from sample to extraction solution; however, the large volume of dispersion solution decreased the concentration of QNs and therefore resulted in low extraction efficiency. The result was shown in Fig. 1e. When the extraction solution volume was $200 \mu \mathrm{L}$, the A values of QNs were the biggest. Therefore, two hundred microliter 1-dodecanol was selected as the extraction solution.

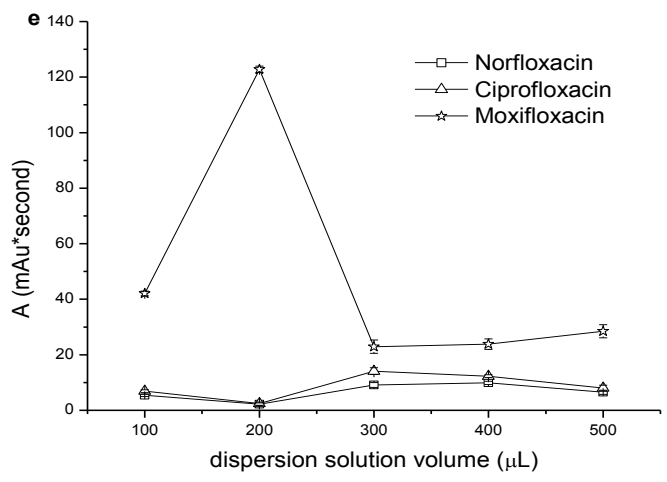

\subsubsection{Effect of ultrasound time}

It is well known that UAE utilized acoustic vibrations to cause cavitation in the liquid, and cavitation enhances the removal of analytes from the matrix to the extraction solution. Therefore, ultrasound can accelerate the QNs transformation process from matrix to extraction solvent. In this work, 5-25 min was optimized; the results were shown in Fig. 1f. It can be seen that the extraction efficiency was best when the time was set at $10 \mathrm{~min}$. So, we selected $10 \mathrm{~min}$ as the optimized ultrasound time. 
Determination of Quinolones in Human Urine and Water Samples by Ultrasound Assisted Dispersive LiquidLiquid Microextraction Based on Solidification of Floating Organic Droplet Followed High Performance Liquid Chromatography

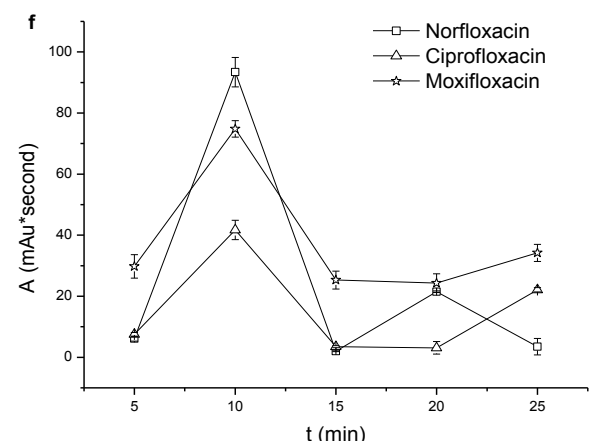

Fig1. Effect of each parameter on extraction efficiency

Therefore, the optimized conditions for UDLLME-SFO extraction of QNs were attained as follows: sample pH 5; extraction solution, $300 \mu \mathrm{L}$ 1-dodecanol; dispersion solution, $200 \mu \mathrm{L} \mathrm{MeOH}$; ultrasound time, $10 \mathrm{~min}$.

\subsection{Analytical Figures of Merit of the UDLLME-SFO-UV Method}

The performance of the UDLLME-SFO-UV method under optimal conditions was investigated. Linear correlation coefficients $(\mathrm{r})$ assessed at six different concentrations were obtained between $\mathrm{A}$ and the corresponding concentrations of QNs in the range of $1-15 \mu \mathrm{g} / \mathrm{mL}$, as shown in Table 1. Limit of detection (LOD) for the QNs, calculated as the analyte concentration for which the value of $\mathrm{A}$ was three times the background noise $(3 \mathrm{~S} / \mathrm{N})$, was $0.03 \mu \mathrm{g} / \mathrm{mL}$ for the HPLC-UV analysis.

Table1. Linear range, slope, intercept, correlation coefficients, and LOD for QNs

\begin{tabular}{|l|l|l|l|l|l|}
\hline QNs & $\begin{array}{l}\text { Linear range } \\
(\mu \mathrm{g} / \mathrm{mL})\end{array}$ & Slope $\pm \mathrm{SD}(\%)$ & Intercept $\pm \mathrm{SD}(\%)$ & $\mathrm{R}^{2}$ & $\mathrm{LOD}(\mu \mathrm{g} / \mathrm{mL})$ \\
\hline CIP & $1-15$ & $2.4128 \pm 0.09$ & $0.0849 \pm 0.01$ & 0.9840 & 0.03 \\
\hline NOR & $1-15$ & $2.507 \pm 0.10$ & $-0.2961 \pm 0.01$ & 0.9740 & 0.03 \\
\hline MOX & $1-15$ & $2.593 \pm 0.08$ & $-0.7372 \pm 0.00$ & 0.9996 & 0.03 \\
\hline
\end{tabular}

\subsection{Determination of QNs in Human Urine and Water Samples}

The developed UDLLME-SFO-HPLC-UV method was further applied to human urine, tap and lake water samples in order to check its practicality. The samples were free with QNs. Recoveries were calculated for the spiked water samples with 2, 5 and $10 \mu \mathrm{g} / \mathrm{mL}$ standards, respectively. The results are listed in Table 2. Satisfactory recoveries were obtained, ranged from $66 \%$ to $102 \%$. This validated the UDLLMESFO-HPLC-UV greatly applicable for the selective extraction, and accurate quantitation of trace QNs in human urine and water samples.

Table2. Recovery of DES from tap and lake water samples

\begin{tabular}{|l|l|l|l|l|}
\hline \multirow{2}{*}{ QNs } & \multirow{2}{*}{ Added $(\mu \mathrm{g} / \mathrm{mL})$} & Recovery \pm SD $(\%)$ & Lake water & Tap water \\
\cline { 2 - 5 } & & Human urine & $73 \pm 8.1$ & $79 \pm 2.0$ \\
\hline \multirow{4}{*}{ CIP } & 2 & $80 \pm 3.9$ & $96 \pm 5.3$ & $97 \pm 4.6$ \\
\cline { 2 - 5 } & 5 & $95 \pm 2.0$ & $92 \pm 6.2$ & $99 \pm 2.9$ \\
\cline { 2 - 5 } & 10 & $102 \pm 2.8$ & $74 \pm 9.5$ & $100 \pm 1.0$ \\
\hline \multirow{3}{*}{ NOR } & 2 & $70 \pm 7.8$ & $88 \pm 8.0$ & $96 \pm 3.8$ \\
\cline { 2 - 5 } & 5 & $85 \pm 5.4$ & $96 \pm 6.1$ & $84 \pm 8.2$ \\
\cline { 2 - 5 } & 10 & $92 \pm 5.0$ & $85 \pm 8.7$ & $72 \pm 8.7$ \\
\hline \multirow{3}{*}{ MOX } & 2 & $66 \pm 9.8$ & $80 \pm 4.8$ & $78 \pm 6.0$ \\
\cline { 2 - 5 } & 5 & $86 \pm 6.2$ & $82 \pm 5.0$ & $73 \pm 7.1$ \\
\cline { 2 - 5 } & 10 & $94 \pm 4.6$ & & \\
\hline
\end{tabular}

\section{CONCLUSION}

In conclusion, a good, easy, and efficient method for the determination of QNs in human urine and water samples was developed. The developed UDLLME-SFO-HPLC-UV offered wide linear range, good 
Determination of Quinolones in Human Urine and Water Samples by Ultrasound Assisted Dispersive LiquidLiquid Microextraction Based on Solidification of Floating Organic Droplet Followed High Performance Liquid Chromatography

quantitative ability, and high precision. Also, this method used microliter extraction and dispersion solutions, was demonstrated to be a cost effective, and eco-friendly option for determination of QNs in water samples.

\section{ACKNOWLEDGMENT}

Financial support from the National Natural Science Foundation of China (81660355), and Research and Training Foundation of Hainan Medical University (HYCX2018003, 201811810003) is gratefully acknowledged.

\section{REFERENCES}

[1] Wang G.N., Yang K., Liu H.Z., Feng M.X. and Wang J.P., Molecularly imprinted polymer-based solid phase extraction combined high performance liquid chromatography for determination of fluoroquinolones in milk, Anal. Method. 8, 5511 (2016).

[2] Sun L., Mei L., Yang H., Zhao K., Li J., Jiang D., Li M. and Deng A, Development and application of immunoaffinity column for the simultaneous determination of norfloxacin, pefloxacin, lomefloxacin, and enrofloxacin in swine and chicken meat samples, Food Anal. Methods 9, 342 (2015).

[3] Leipert J., Bobis I., Schubert S., Fickenscher H., Leippe M.and Tholey A. Miniaturized dispersive liquid-liquid microextraction and MALDI MS using ionic liquid matrices for the detection of bacterial communication molecules and virulence factors, Anal. Bioanal. Chem 410, 4737 (2018).

[4] Guidi L. R., Santos F. A., Ribeiro A. C., Fernandes C., Silva L. H. and Gloria M. B., A simple, fast and sensitive screening LC-ESI-MS/MS method for antibiotics in fish, Talanta 163, 85 (2017).

[5] X. Yu, H. Liu, C. Pu, J. Chen, Y. Sun and L. Hu. Determination of multiple antibiotics in leafy vegetables using QuEChERS-UHPLC-MS/MS, J. Sep. Sci. 41, 713 (2018).

[6] Jansomboon W., Boontanon S. K., Boontanon N. and Polprasert C, Determination and health risk assessment of enrofloxacin, flumequine and sulfamethoxazole in imported pangasius catfish products in Thailand, J. Environ. Sci. Health. B 53, 108 (2018).

[7] Rodriguez-Gomez R., Garcia-Corcoles M. T., Cipa M., Barron D., Navalon A. and Zafra-Gomez A., Determination of quinolone residues in raw cow milk. Application of polar stir-bars and ultra-high performance liquid chromatography-tandem mass spectrometry, Food Addit. Contam. Part A Chem. Anal. Control. Expo. Risk Assess., 35, 1127 (2018).

[8] Wen Y., Li J., Zhang W. and Chen L., Dispersive liquid-liquid microextraction coupled with capillary electrophoresis for simultaneous determination of sulfonamides with the aid of experimental design, Electrophoresis 32, 2131 (2011).

[9] Niu Z., and Wen Y., Determination of diethylstilbestrol in water samples by dispersive liquid-liquid microextraction based on solidification of floating organic droplet followed UV spectrometry, International Journal of Advanced Research in Chemical Science, 5, 1(2018).

[10] Niu Z., Zhang J. and Wu D., Wen Y., Dispersive liquid-liquid microextraction with solidification of floating organic drop combined with high performance liquid chromatography for the determination of phenolic oestrogens from human urine and water samples, Int. J. Environ. Anal. Chem., 99, 444 (2019).

[11] Lin C., Deng Y., Liao W., Sun S., Lin W., Chen C., Electrophoretic behavior and pKa determination of quinolones with a piperazinyl substituent by capillary zone electrophoresis, J. Chromatogr., A, 1051, 283 (2004).

Citation: Yingying Wen, et.al. (2019). "Department of Environmental Science, School of Tropical and Laboratory Medicine, Hainan Medical University, Haikou 571199, China". International Journal of Advanced Research in Chemical Science (IJARCS), 6(5), pp. 13-18, DOI:http://dx.doi.org/10.20431/23490403.0605003.

Copyright: () 2019 Authors. This is an open-access article distributed under the terms of the Creative Commons Attribution License, which permits unrestricted use, distribution, and reproduction in any medium, provided the original author and source are credited. 